\title{
LES CALCULS STRATEGIQUES DERRIERE LE "CANADA BILL"
}

\section{Daniel Latouche*}

The author views the 1982 Canada Bill as yet another attempt to "normalize" Quebec. He analyzes the forms of this political normalization and reflects upon the fact that the Constitutional Conference was actually a negotiation between Quebec and English Canada. He then moves on to the three other objectives of the Constitutional Conference, which were the repatriation of the constitution, a constitutional reform, and the political defeat of the "Parti Québécois." The author concludes that Quebec will have to use other avenues to achieve the political equality it still aspires to. Up until now Quebec thought equality could be achieved within Canada through renewed federalism, or with Canada through sovereignty-association. Now Quebec may consider that equality will be achieved against Canada through independence.

\section{INTRODUCTION}

Ce n'est pas faire accroc au discours scientifique que d'affirmer qu'on assiste depuis mai 1980 à une tentative de normalisation politique du Québec dont le Canada Bill de 1982 n'est qu'une étape. A cause d'une association avec la Tchécoslovaquie de 1968 ou la Pologne de 1983 une telle affirmation en choquera plusieurs. Pourtant les effets attendus sont les mêmes. Il s'agit, pour Ottawa et les autres gouvernements provinciaux, de mettre un terme une fois pour toutes aux velléités d'autonomie d'une société québécoise qui dérange passablement ce qui est devenu le projet politique canadien par excellence, soit la dépendance économique tranquille du Canada envers les Etats-Unis.

Elles furent rares au Québec français les manifestations, que ce soit sous forme de colloques universitaires ou de parades royales, organisées pour marquer la proclamation de ce Canada Bill. Que ce sentiment de dépit soit partagé à la fois par les partisans de la souveraineté et ceux du fédéralisme témoigne de la profondeur avec laquelle est ressenti ce sentiment d'échec. Pour les deux groupes, cet accord constitutionnel marque la fin du rêve qu'ils partageaient: celui de reconstruire le Canada afin d'assurer au Québec l'égalité politique qui le ferait sortir une fois pour toutes de son statut de collectivité minoritaire. Pour les souverainistes, l'accord constitutionnel, non pas tant par son contenu que par le rapport de forces et le processus qui l'a engendré, marque l'échec de l'idée même de souverainetéassociation. Pour les fédéralistes, c'est la fin du projet d'un fédéralisme renouvelé 
en profondeur. A brève échéance, Claude Ryan, le chef du Parti Libéral du Québec, qui incarnait cette vision, en sera la victime.

Le Québec avait beaucoup à gagner et beaucoup à perdre dans cette négociation avec le reste du pays. Plusieurs stratégies auront été employées et toutes auront échoué. Jusqu'en 1976, c'est la stratégie des accommodements qui a prévalu. On a tenté d'aller chercher le plus grand nombre de pouvoirs législatifs, de responsabilités administratives et de ressources fiscales, bref, tout ce qui pouvait représenter un acquis pour le Québec. En 1976, le gouvernement du Parti Québécois effectua un virage de $180^{\circ}$. Fini la stratégie minimale des accommodements, la stratégie maximale allait réussir là où celle des "petits pas" avait échoué.

Cette stratégie, elle aussi, a échoué et, avant de songer à remettre le navire à flot, il faut évaluer correctement les dommages. Pour l'instant, ces dommages ont nom: normalisation politique, déstabilisation sociale et étranglement économique. Les grêves dans le secteur de l'éducation, des affaires sociales et de la fonction publique qui ont déchiré le Québec à l'hiver 1982 ne sont qu'un signe avantcoureur d'une situation qui risque fort de se généraliser. N'étant plus un bien de pouvoir, la scène québécoise va devenir le terrain privilégié de ces affrontements stériles.

La normalisation politique touche trois aspects de l'échiquier québécois: le déplacement vers Ottawa de centres de décision qui auparavant appartenaient en exclusivité à l'Assemblée nationale, la volonté d'éliminer électoralement le Parti Québécois et la vassalisation definitive du Parti Libéral du Québec.

Le texte qui suit est une évaluation des formes que prend cette normalisation ainsi qu'une réflexion sur ce qui fût sans aucun doute la principale erreur des élites québécoises, à savoir que la négociation constitutionnelle était en fait une négociation entre le Québec et le Canada anglais.

\section{II}

\section{L'ObJectif Stratégique: la Normalisation Politique du QUÉBEC}

Ainsi donc, il n'y aura pas de véritable nouvelle entente politique au Canada. Que cette possibilité ait pu miroiter si longtemps aux yeux des élites politiques québécoises est certes le signe d'un espoir largement partagé, mais aussi d'une erreur d'appréciation.

Dans une unanimité touchante, les élus canadiens-français en poste à Ottawa et les "observateurs-canadiens-anglais-sympathiques-à-la-cause-québécoise" affirment maintenant que rien n'est terminé, que le rapatriement et l'accord sur la formule d'amendement rendent maintenant possible une véritable réforme constitutionnelle, etc. ${ }^{1}$ Doit-on considérer ces propos comme les manifestations d'une

1. De telles opinions sont fréquemment exprimées dans plusieurs revues canadiennes anglaises dont The Magazine, Canada Dimension, Options, et Canadian Forum. En février 1983 le solliciteur général de l'Ontario, M. Roy McMurty, déclarait devant les étudiants de l'Université Laval qu'il fallait peut-être envisager de modifier la constitution pour accorder au Québec un droit de veto sur les questions culturelles. Robert Stanfield, l'ancien chef du Parti Conservateur du Canada, a très bien resumé cette "nouvelle" attitude dans What to Do about Quebec's Isolation, 3 Policy Options 6 (1982). 
bonne foi culpabilisée ou d'un paternalisme éhonté? Difficile à dire. Chose certaine, c'est considérer les Québécois comme une quantité politique bien négligeable que de les noyer ainsi sous ces propos faussement réconfortants. Cette incapacité du reste due Canada d'admettre ouvertement que le Canada Bill constitue ni plus ni moins qu'une "raclée constitutionnelle" infligée au Québec, témoigne à sa façon du manque de maturité politique qui ne veut même pas reconnaître ses victoires.

Pourtant une défaite est une défaite. Si un jour, elle doit être suivie d'une victoire pour le Québec, cela dépend davantage d'une évaluation sérieuse des erreurs passées que de bons mots des adversaires. En politique, comme au cinéma d'ailleurs, les "bons" ne gagnent pas toujours.

Que le gouvernement fédéral, les élites politiques canadiennes-anglaises, l'opinion publique et les intellectuels considèrent le Canada Bill comme une grande victoire, une manifestation du génie canadien ou simplement une étape importante dans le développement politique du pays, illustre mieux que tous les propos du plus biaisé des séparatistes la faillite du fédéralisme canadien.

Avec les Iles Tonga et la République Centre-africaine de l'empereur Bokassa, le Canada est le seul pays qui a cru bon réaffirmer récemment le caractère monarchique de ses institutions centrales. Que cette réaffirmation soit le fait de Sa Majesté elle-même dans le cadre d'un spectacle digne d'un Disneyland politique, fait ressortir pleinement la naiveté de tous ces Québécois, qui pendant des années ont pris pour acquis que le Canada se donnerait un nouveau cadre politique.

Il ne reste plus qu'à espérer que la réforme constitutionnelle n'ira pas plus loin. Le ridicule risquerait alors d'achever ce que ce pays conserve encore de dignité. Espérons aussi qu'un peu de "self-respect" bien placé fera réaliser au reste du pays que maintenant que le Québec a été normalisé, ce serait ajouter l'injure à l'insulte de lui offrir, comme on l'a fait pour les handicapés et qu'on s'apprête à le faire pour les autochtones, des garanties de protection pour sa culture et sa langue. A défaut de l'égalité politique, mieux vaut tout de même être une province comme les autres qu'une réserve culturelle qui viendrait témoigner de ce que le Canada aurait pu être.

D'ailleurs s'il fallait qu'une telle négociation reprenne, il est à prévoir que le Québec y perdrait encore quelques plumes. A-t-on dejâ vu un négociateur réussir à obtenir davantage lorsqu'il est en position d'extrème faiblesse. Que l'on discute depuis quelque temps à Ottawa de la possibilité d'accorder au Québec un droit de veto partiel avec compensation financière pour ce qui est des questions de culture, de langue, de droit civil et d'éducation vient confirmer que contrairement à l'esprit et à la lettre de l'entente de 1867, il est maintenant possible à la majorité politique du pays de modifier les équilibres régissant ces secteurs sans que le gouvernement de la province de Québec ne puisse y faire quoi que ce soit. Si tel n'était pas le cas, on ne parlerait pas ainsi des garanties constitutionnelles, dont on conditionne d'ailleurs le don à une bonne conduite des Québécois et de leur gouvernement. Le mépris a toujours plus d'un temps.

Chez ceux-là même qui ont imaginé et imposé ce Canada Bill, on ne fait plus confiance au jeu naturel des institutions politiques qui ont toujours été au coeur du 
fédéralisme canadien et qui ont su protéger adéquatement la plus importante minorité culturelle du pays. Après s'être assuré que cette minorité demeurerait une minorité, voilà qu'on s'inquiète de ce que la majorité purrait lui imposer. Ne dirait-on pas que la majorité anglophone du pays cherche à s'empêcher constitutionnellement de poser des gestes qu'elle pourrait être tentée de poser politiquement. Par la façon dont il a été imposé et par son contenu, ce Canada Bill vient confirmer ce que d'aucuns soupçonnaient et d'autres espéraient: le gouvernement et les institutions politiques du Québec ne sont plus des gardiens très solides de la spécificité française du Québec. Celle-ci est devenue maintenant l'affaire de tout le Canada. Même pour ce qui est de la culture, de la langue et de l'éducation, on cherche à faire du gouvernement québécois l'agent d'exécution de politiques décidées à Ottawa. La culture si importante, que tous les gouvernements du Canada, ceux de l'Alberta, du Yukon et d'Ottawa y compris, veulent mainenant s'en mêler. Jamais on aura tant "aimé" les Québécois. ${ }^{2}$ Leur culture est même jugée trop essentielle pour leur en accorder la gestion exclusive.

S'étant rendu compte que l'affirmation culturelle et linguistique transcendait au Québec les lignes partisanes traditionnelles, on a voulu et on a réussi à les soustraire à l'exercice du champ politique québécois. Même les liberaux de Claude Ryan sont suspects. Ne sont-ils pas en danger eux aussi de succomber à la tyrannie d'une majorité électorale très satisfaite des lois linguistiques actuelles? C'est à des tribunaux dont les juges sont entièrement nommés par le gouvernement central, qu'il appartiendra maintenant de corriger les erreurs de parcours de la démocratie électorale québécoise. Il ne manque plus que la nomination par Ottawa d'un gauleiter en charge du Québec et la normalisation serait complète. Ce n'est pas une coïncidence si cette normalisation politique du Québec survient au moment même où la première génération québécoise née aprés 1960 fait son irruption sur la scène économique et politique. ${ }^{3}$ Certes on se dit très content de voir cette génération manifester le sens et le goût des affaires, ainsi qu'une volonté de ne pas se contenter du Québec et des institutions canadiennes-françaises traditionnelles. On veut que cette génération se lance à l'assaut du Canada, mais pas à partir d'une situation d'égalité politique. C'est le propre de toute majorité d'aimer que ses minorités soient dynamiques et pittoresques, mais qu'elles demeurent avant tout des minorités.

A partir de la perspective peu suspecte de collectivisme et qui n'est pas sans rappeler les nouveaux économistes du "supply side," J.-L. Migué a montré com-

2. De nombreuses provinces (Alberta, Ontario, Saskatchewan, Manitoba, Nouveau-Brunswick) ont récemment mis sur pied des programmes culturels spéciaux pour leur minorités francophones, motivés en bonne partie par une volonté de faire échec à la prétention du gouvernement fédéral que c'était là l'une des responsabilités particulières du gouvernement "national." Nul doute que les tentatives anterièures du gouvernement du Québec, notamment au moment du référendum de 1980, d'obtenir l'appui de ces communautés, a aussi été un facteur déterminant dans ce renversement d'attitude face à la culture française hors-Québec.

3. Le groupe des 30-40 (en 1983) n'a été que faiblement marqué par la période Duplessiste. Très nationaliste, mais d'un nationalisme davantage politique et économique que culturel, ce groupe constitue ce qu'il est convenu d'appeler la nouvelle élite. Voir à ce sujet D. CLIFT, LE DEcLin du NATIONALISME AU QUÉBEC (1981); Crete \& Landry, Vieillesse québécoise et changement politique 1960 à 1980, 6 ANTHROPOLOGIE ET SOCIÉTÉs 45 (1982). 
ment la langue, les réseaux culturels et même le nationalisme pouvaient être des instruments fort rentables de promotion économique pour les élites managérielles québécoises. Parlant de la survie de la société québécoise, il écrit à propos de la politique d'accommodement de Robert Bourassa:

La politique libérale mène tout droit à l'effritement progressif des institutions québécoises et à l'intégration certaine, sinon lucide, des Québécois au réseau d'institutions le plus reluisant. Enfin la survie d'une société québécoise n'est compatible qu'avec le renforcement de ses propres institutions, c'est-à-dire avec l'institutionnalisation de ses relations avec la firme plurinationale. A son tour le processus ne peut s'implanter qu'avec la francisation de tous les échelons de la hiérarchie technique et administrative de la firme. ${ }^{4}$

C'est cette rentabilisation du nationalisme québécois que le Canada Bill veut à tout prix rompre, afin que même au Québec les jeunes managers francophones soit désavantagés par leur appartenance culturelle. Les tentatives de l'automne 1982 du gouvernement fédéral d'éliminer de la carte du ciel la compagnie Québecair, celle encore plus visible de limiter l'expansion de la Caisse de dépôts et de placements du Québec par le projet de loi C-31 en sont les exemples les plus récents. ${ }^{5}$

Encore une fois, on les aime tellement ces Québécois qu'on voudrait qu'ils se distribuent partout au Canada. A chaque province, ses petits immigrants québécois, mais des Québécois pour qui le Québec n'est qu'une province d'origine et non pas leur espace politique privilégié. Ce qu'on veut surtout préserver c'est une culture canadienne-française minoritaire, après tout n'est-il pas vrai que ce sont les minorités culturelles qui ont les cultures les plus dynamiques. Il faut donc protéger les Québécois contre la stérilité qui découlerait de leur statut de majoritaires. Nous l'avons échappé belle.

Les plus forts applaudissements au Canada Bill proviennent de la minorité anglophone du Québec. Cela aussi est fort significatif. Que sur une question qui a fait l'unanimité de toutes les fractions politiques du Québec français, il n'est pas trouvé un seul groupe de Québécois anglophones pour s'opposer à un Canada Bill qui réduit les pouvoirs de la communauté politique à laquelle ils ne cessent de proclamer leur appartenance, met en évidence toute l'hypocrisie de ces hauts cris.

L'appartenance des Canadiens anglais du Québec à la communauté politique du Québec n'est possible à leurs yeux que si le cadre politique fédéral impose au Québec un statut de minorité permanente à l'intèrieur du Canada. Pour les anglophones du Québec, l'existence d'un Canada français encadré et protégé légitimise leur participation à ce qu'ils considèrent être la véritable majorité du pays, la majorité anglophone, tout en leur permettant d'échapper au statut de minorité culturelle que l'actuel gouvernement du Québec voudrait leur imposer. Si les anglophones du Québec sont pour être une minorité au Québec, alors les Québécois vont aussi l'être à l'échelle du pays. A minorité, minorité et demie.

L'intégration des anglophones du Québec à la vie collective est un mythe qu'il

4. Migué, La nationalisme québécois, le développement économique et la théorie économique de l'information, dans Le Système POlitique Québécois 449 (E. Cloutier et D. Latouche eds. 1979).

5. Ce projet de loi non encore adopté par le Parlement fédéral prévoit qu'une institution étatique provinciale ne pourra plus désormais détenir plus de $10 \%$ des intérêts d'une entreprise impliquée dans le transport inter-provincial. Cette mesure vise essentiellement à empêcher la Caisse des dépöts d'acquérir une participation trop importante dans la multinationale Canadian Pacific. 
est temps de dénoncer. Certes, lorsqu'on leur demande s'ils sont bilingues, les anglophones du Québec ont de plus en plus tendance à répondre par l'affirmative ${ }^{6}$ mais une majorité absolue de ceux-ci refuse encore de reconnaître que le français doit être la langue majoritaire au Québec. ${ }^{7}$ Les récentes côtes d'écoute des stations de radio et de télévision montréalaises ont montré que l'écoute des médias francophones par les anglophones est tellement insignifiante qu'elle ne peut être comptabilisée. $^{8}$ Il suffit de travailler dans un milieu anglophone comme l'Université McGill pour mesurer le peu d'intégration des jeunes anglophones à la vie culturelle française qui les entoure. A ce sujet, le contraste avec les jeunes francophones est étonnant. ${ }^{9}$

D'ailleurs l'étude récente de T.R. Balakrishnan vient de confirmer que c'est à Montreal que la ségrégation ethnique residentielle est la plus élevée de toutes les villes canadiennes, surtout pour ce qui est de l'opposition entre d'un côté le groupe ethnique français et de l'autre tous les autres groupes (les taux de ségrégation residentielle entre les groupes ethniques non-français sont beaucoup plus faibles). De plus, cette ségrégation a augmenté entre 1961 et 1971 et demeure relativement insensible à la distribution du statut socio-économique. ${ }^{10}$

Quelques questions posées lors de différents sondages révèlent que cette faible intégration des anglophones du Québec est aussi le fait des nouvelles générations. (Voir Tableau 1.) A peine 5\% s'identifient à la Fête nationale québécoise du 24 juin ou se definissent comme des Québécois. Moins de $20 \%$ d'entre eux s'identifient prioritairement au gouvernement du Québec plutôt qu'a celui d'Ottawa.

\section{TABleaU 1}

IDENTIFICATION NATIONALE DES QUÉbÉCOIS ANGLOPHONES ET FRANCOPHONES

\begin{tabular}{|c|c|c|c|c|c|c|}
\hline & & Total & 18-24 & 25-34 & 35.44 & 45 et plus \\
\hline $\begin{array}{l}\text { Identification prioritaire } \\
\text { au gouvernement du } \\
\text { Québec }\end{array}$ & $\begin{array}{l}\text { anglo } \\
\text { franco }\end{array}$ & $\begin{array}{l}19 \% \\
53 \%\end{array}$ & $\begin{array}{l}21 \% \\
70 \%\end{array}$ & $\begin{array}{l}18 \% \\
63 \%\end{array}$ & $\begin{array}{l}21 \% \\
50 \%\end{array}$ & $\begin{array}{l}17 \% \\
40 \%\end{array}$ \\
\hline $\begin{array}{l}\text { Identification au } 24 \\
\text { juin plutôt qu'au ler } \\
\text { juillet }\end{array}$ & $\begin{array}{l}\text { anglo } \\
\text { franco }\end{array}$ & $\begin{array}{r}5 \% \\
55 \%\end{array}$ & $\begin{array}{r}0 \% \\
60 \%\end{array}$ & $\begin{array}{r}8 \% \\
62 \%\end{array}$ & $\begin{array}{r}0 \% \\
47 \%\end{array}$ & $\begin{array}{r}8 \% \\
32 \%\end{array}$ \\
\hline $\begin{array}{l}\text { Identification comme } \\
\text { Québécois }\end{array}$ & $\begin{array}{l}\text { anglo } \\
\text { franco }\end{array}$ & $\begin{array}{r}5 \% \\
38 \%\end{array}$ & $\begin{array}{l}13 \% \\
55 \%\end{array}$ & $\begin{array}{r}8 \% \\
49 \%\end{array}$ & $\begin{array}{r}1 \% \\
31 \%\end{array}$ & $\begin{array}{r}1 \% \\
25 \%\end{array}$ \\
\hline
\end{tabular}

6. Sondage Crop-CBC, février 1982.

7. D'ailleurs on peut s'interroger sur la validité scientifique de demander directement, sans aucun contrôle ou définition préalable, à des gens s'ils sont bilingues ou non, alors qu'on sait que la très grande majorité de ceux-ci estiment qu'ils devraient l'être.

8. Sauf pour ces soirées ou un match de hockey est présenté en exclusivité à une station de télévision francophone.

9. Voir à ce sujet, P. Georgeault, Conscience Linguistique des Jeunes Québécois (1981).

10. Balakrishnan, Changing Residential Segregation in Metropolitan Areas of Canada, 19 CAN. REV. OF SOCIOLOGY AND ANTHROPOLOGY 92 (1982). 
Ces chiffres ne trompent pas. Depuis peu, les anglophones du Québec se sont donnés une association, Alliance Québec, dont on a vanté l'ouverture d'esprit et la modération parce qu'elle reconnaissait que les anglophones du Québec veulent vivre au Québec. Mais lorsque vient le temps de discuter des modalités de ce vouloir vivre, on se rend compte qu'ils veulent vivre exclusivement dans leur langue, dans leurs institutions, avec leurs structures. Certes, on admet le droit des $80 \%$ de francophones de vivre en français mais c'est une admission du bout-deslèvres dictée par l'évidence de la situation. Ce qu'on espère obtenir, c'est le retour au bilinguisme d'antan, c'est-à-dire des francophones bilingues et des anglophones qu'on va cesser d'inquiéter à cause de leur unilinguisme.

Cet appui massif du groupe anglophone québécois au Canada Bill est une erreur qui risque rapidement de se retourner contre cette communauté. Plus grande est l'insécurité dans laquelle on place la culture française, plus grandes sont les menaces dirigées contre l'autonomie des institutions politiques québécoises, plus contraignantes risquent d'être les politiques linguistiques du gouvernement québécois. A court terme, il peut se révéler fort rentable pour ce groupe de devenir le pupille du gouvernement fédéral mais en se faisant ainsi l'allié objectif de ceux qui veulent normaliser le Québec, ces anglophones risquent de se placer euxmêmes dans la situation peu enviable de devenir rapidement "expandable" politiquement. C'est ce qui est survenu aux communautés françaises hors Québec que le gouvernement central utilise maintenant comme autant de parcs d'exhibition pour sa politique linguistique. La décision prise à l'hiver 1981 par six Secrétariats d'Etat de remplacer ses subventions directes à l'Association canadienne-française d'Ontario par des subventions particulières à chaque organisme est un avant goût de cette nouvelle situation.

Cette volonté stratégique de remettre le Québec à sa place politiquement apres vingt ans de grignotage et de menaces a connu depuis le référendum de mai 1980 une accélération sans précédent. Ce fut d'abord la course contre la montre pour terminer l'opération avant l'arrivée au pouvoir du Parti Libéral du Québec dont les propositions de révision constitutionelle étaient jugées inacceptables par le gouvernement fédéral. Cette dernière manoeuvre a echoué à moitié. La conférence constitutionnelle de septembre 1980 n'a certes pas abouti mais le Parti Libéral de Claude Ryan a quant à lui été rejeté électoralement. Une toute nouvelle situation stratégique s'ouvrait alors aux négociateurs fédéraux et à ceux des provinces anglophones. C'est ce que nous voudrions montrer dans la section suivante.

III

\section{Les Opérations sur le Terrain la Conférence et le Canada BILL}

Probablement parce qu'ils sont aussi habitués aux conférences constitutionnelles qu'au retour de l'hiver, peu d'observateurs ont compris que la conférence de novembre 1981 ne pouvait pas aboutir de la même façon que les autres. Certes tous les ingrédients d'une conférence-comme-les-autres étaient présents: Front Commun de certaines provinces, menaces de dissension dans le Front Commun, dévoilement dans les journaux de documents hautement confidentiels, déclarations 
à l'effet qu'il s'agit de la conférence de la dernière chance, cynisme de la part du Québec, couverture exagérée par les médias. Ce qui était différent, c'est que pour la première fois, le Canada-anglais par la voix de ses premiers ministres, ainsi que le gouvernement fédéral avaient enfin la possibilité d'atteindre conjointement quatre objectifs qu'ils chérissaient individuellement depuis longtemps: (1) faire subir un échec politique de première importance au gouvernement du Parti Québécois, (2) isoler le Québec et mettre un terme à toute velléité de particularisation politique de cette province, que ce soit sous la forme de statut particulier, de fédéralisme asymetrique, d'états-associés ou de souveraineté-association, (3) rapatrier le document constitutionnel et ainsi satisfaire P.E. Trudeau et (4) réaliser une réforme constitutionnelle optimale, c'est-à-dire obtenir un accord sur le maximum de points qui mettraient en cause le minimum de changement des équilibres fondamentaux régissant la fédération.

On aura remarqué que cette façon d'envisager le processus de négociation constitutionnelle s'apparente au modèle III de G.T. Allison dans Essence of Decision. ${ }^{11}$ Certes, l'étude Allison porte sur un sujet, la crise des missiles cubains de 1962, qui est fort éloigné de la question constitutionnelle canadienne, mais le cadre théorique demeure fort éclairant. Allison propose trois modèles pour expliquer une décision politique.

Le modèle rationnel privilégie l'existence d'un acteur unique qui déplace ses pions au gré des stratégies et tactiques qu'il aura élaborées. L'approche organisationnelle présume plutôt que le décideur n'est pas une entité unique, mais une alliance flottante entre des organisations semi-autonomes, chacune avancant ses pièces selon un code opérationnel précis. Quant au troisième modèle, il implique l'existence d'un nombre distinct de joueurs, chacun avec ses objectifs particuliers, mais qui partagent-certains étant plus importants que d'autres-la décision sur l'ensemble du jeu. Alors que dans le modèle I la décision politique est la résultante d'une évaluation coûts-bénéfices, le modèle II suppose au contraire la primauté d'une sorte de code de procédures dérivé d'un processus d'apprentissage organisationnel, tandis que le dernier modèle privilégie les relations de négociations et d'échanges entre les acteurs d'un même camp. Dans une métaphore devenue célèbre Allison caractérise ainsi ces trois modèles:

A central metaphor illuminates the differences among these models. Foreign policy has often been compared to moves and sequences of moves in the game of chess. Imagine a chess game in which the observer could see only a screen upon which moves in the game were projected, with no information about how the pieces came to be moved. Initially, most observers would assume-as Model I does-that an individual chess player was moving the pieces with reference to plans and tactics toward the goal of winning the game. But a pattern of moves can be imagined that would lead some observers, after watching several games, to consider a Model II assumption: the chess player might not be a single individual but rather a loose alliance of semi-independent organizations, each of which moved its set of pieces according to standard operating procedures. For example, movement of separate sets of pieces might proceed in turn, each according to a routine, the king's rook, bishop, and their pawns repeatedly attacking the opponent according to a fixed plan. It is conceivable, furthermore, that the pattern of play might suggest to an observer a Model III assumption: a number of distinct players, with distinct objectives but shared

11. G. Allison, EsSence of Decision (1971). 
power over the pieces, could be determining the moves as the resultant of collegial bargaining. For example, the black rook's move might contribute to the loss of a black knight with no comparable gains for the black team, but with the black rook becoming the principal guardian of the palace on that side of the board. ${ }^{12}$

Mais revenons à notre constitution. Chacun des quatre objectifs identifiés plus haut peut être considéré comme l'objectif principal des quatre principaux acteurs qui formaient ce que nous appellerons le camp "canadien" (par opposition au camp québécois).

- rapatriement de la constitution: c'est l'objectif personnel prioritaire de Pierre E. Trudeau

- réforme constitutionnelle optimale: c'est l'objectif des neuf premiers ministres des provinces anglophones

- normalisation politique du Québec: l'objectif de l'appareil politique bureaucratique du gouvernement central

- défaite sérieuse du Parti Québécois: l’objectif de la députation canadienne-française à Ottawa

Nous faisons l'hypothèse que l'accord constitutionnel de novembre 1981 a été rendu possible parce qu'il rencontrait ces quatre objectifs. Il a certes fallu une bonne dose de négociations, de coordination forcée, de simple hasard et d'erreurs de l'adversaire québécois pour arriver à une entente, mais ce qu'il importe de constater c'est que pour la première fois ces quatre acteurs ont réussi à en arriver à un accord dont les principaux perdants_et là-dessus les deux camps sont d'accordsont les antithèses québécoises des acteurs mentionnés plus haut, soit René Lévesque (P.E. Trudeau), la communauté politique du Québec (Canada anglais), le gouvernement et l'appareil étatique québécois (gouvernement central), le Parti Québécois (Parti Libéral fédéral).

Quatre acteurs, avons-nous dit, mais aussi deux camps. En effet, jamais une conférence constitutionnelle n'aura si ouvertement mis en presence deux adversaires. Alors que la conférence s'est officiellement ouverte avec d'un côté, le camp du gouvernement central et ses deux alliés provinciaux et de l'autre, celui des huit provinces récalcitrantes, elle est rapidement devenue, au niveau de la pratique des négociations tout au moins, un affrontement entre le Québec et le reste du pays. Les représentants des autres provinces et ceux du gouvernement central auront beau proclamer leur innocence à ce sujet, les commentateurs et observateurs canadiens-anglais insister sur le jeu normal du "give-and-take" constitutionnel, ce n'est pas ainsi que les choses se sont passées. On peut certes reprocher aux négociateurs québécois de s'être fait joués comme autant de lapins mais si c'est le cas, il faut admettre aussi la contrepartie: ils avaient en face d'eux un groupe décidé de renards et de loups pour qui des accords signés et des paroles données ne voulaient rien dire. Si effectivement les Canadiens anglais sont fiers de la façon dont cette conférence s'est déroulée-rencontres secrètes dans la nuit où le Québec n'était pas invité, manoeuvres et chantages_cela en dit beaucoup sur le genre de pays auquel ils aspirent.

12. Id. at 7 . 
Il faudrait aussi rejeter une fois pour toutes l'explication qui veut que l'actuel gouvernement du Québec ait été décidé et surtout incapable de signer le moindre accord constitutionnel avec ses partenaires canadiens. D'ou son isolement à la conférence de novembre 1981. On reste bouche bée devant le mélange d'incompréhension du jeu politique et de mépris anti-québécois qu'un tel jugement suppose.

On mesure mieux aujourd'hui comment le gouvernement du Parti Québécois avait besoin d'un accord constitutionnel qui aurait englobé un rapatriement, une formule d'amendement, une charte des droits et une liste de grands principes. Il n'y a qu'à voir la dégringolade que ce gouvernement a subie dans l'opinion publique, les démissions qui l'affligent, les dissensions qui le déchirent, les défaites aux elections partielles et son état général de désarroi pour comprendre comment un accord aurait pu lui être bénéfique. Depuis sa ré-élection en avril 1981 le gouvernement du Parti Québécois a vu quatre de ses ministres démissionner: Claude Morin, Denis de Belleval, Claude Charron et Lucien Lessard, sans compter le président de l'Assemblée nationale, Claude Vaillancourt. Aujourd'hui, si un accord acceptable au Québec avait pu être signé, plus personne ne parlerait de cet accord et le débat aurait repris de plus belle sur le seul terrain où le gouvernement du Parti Québécois pouvait espérer jouer gagnant: celui du partage des pouvoirs.

Il faut accorder à l'actuel gouvernement du Québec un minimum d'intelligence politique. Toutes ces choses étaient connues. Certes, elles n'étaient pas appréciées par tous, mais les sondages internes menés par le Parti Québécois avaient facilement eu raison des récalcitrants: un accord constitutionnel était un objectif majeur du gouvernement québécois. Depuis 1975 le Parti Québécois effectue régulièrement des sondages d'opinion dont les résultats sont confidentiels mais auxquels l'auteur a pu avoir accés en tant qu'ancien membre du Bureau du premier ministre. L'un des derniers sondages publics et qui illustre bien la baisse de popularité du Parti Québécois est celui d'octobre 1982 du Centre de sondage de l'Université de Montréal (rapporté dans la Presse, 16 décembre 1982, p. B.7) et qui donne $42 \%$ des intentions de vote au Parti Québécois contre $51 \%$ au Parti Libéral et $7 \%$ aux autres partis. Parmi les autres sondages publics qui traitent des mêmes questions, mentionnons celui de la firme SORECOM (Le Devoir, 5 octobre 1981) et du Centre de sondage de l'Université de Montréal (Le Devoir, 20 janvier 1982). Ce calcul stratégique était tellement évident-trop peut-être-que le gouvernement québécois a accepté avant même que ne débute la conférence, davantage de compromis que l'ensemble des gouvernements québécois ne l'avaient fait avant lui. La liste de ces compromis "historiques" acceptés par le gouvernement Lévesque a probablement du faire frémir les Lesage, Johnson, Bertrand et Bourassa.

- áccord sur le principe et les modalités du rapatriement

- accord sur une formule d'amendement

- accord sur la possibilité de rapatrier la constitution avant tout accord sur le partage des pouvoirs

- accord sur une Charte des droits y compris les droits linguistiques

- accord sur les principes directeurs de la fédération canadienne 
- accord sur la place et la définition officielle du Québec dans un nouvel accord constitutionnel

- accord sur un calendrier de négociations futures.

Affirmer, à partir de ces faits et de l'entente signée entre le Québec et sept autres provinces, que le gouvernement du Québec n'aurait pas signé une entente constitutionnelle, revient à dire que l'actuel gouvernement du Québec est illégitime et qu'il ne respecte pas ses ententes parce que son objectif à long terme est la souveraineté du Québec. C'est là une insulte dont le caractère raciste n'aura pas échappé à tous les "Frenchies" du Québec: les $50 \%$ qui ont voté oui au référendum, ceux qui ont voté non en pensant que cela équivalait à une nouvelle constitution, les $60 \%$ qui ont appuyé le Parti Québécois lors du scrutin d'avril 1981 et tous ceux qui ont voté pour Claude Ryan en pensant que le Parti Libéral était en meilleure position pour négocier un accord qui reconnaisse les droits légitimes du Québec.

Le plus étonnant, ce n'est peut-être pas qu'on retrouve un tel argument dans la bouche des négociateurs fédéraux et chez ceux des provinces anglophones, mais qu'aujourd'hui bon nombre d'intellectuels canadiens-anglais acceptent de se prêter à cette entreprise de destabilisation politique.

C'est parce qu'ils avaient fait sensiblement la même analyse que les négociateurs québécois-a savoir qu'un accord constitutionnel favoriserait plutôt qu'il ne nuirait au gouvernement péquiste —que les négociateurs fédéraux et ceux des autres provinces ont jugé préférable de proposer un accord inacceptable au Québec. C'est le jeu normal des négociations: des que vous avez trouvé l'objectif minimal de l'adversaire, il faut tout mettre en oeuvre pour l'empêcher d'y arriver. La signature d'une entente aurait representé une victoire stratégique importante pour le Québec, car elle aurait effacé le stigma d'un gouvernement peu intéressé à la réforme constitutionnelle, alors qu'elle aurait placé le Parti Libéral de Claude Ryan dans une situation intenable: celle de s'opposer à un accord négocié par le P.Q. sous prétexte qu'il est fondé sur trop de compromis! La constitution rapatriée, la formule d'amendement adoptée et la charte des droits enchassée, toutes ces manoeuvres qu'on avait si souvent utilisées pour retarder la négociation véritable, auraient finalement été reglées.

Si on envisage maintenant la situation du point de vue du gouvernement fédéral et de celui des neuf autres gouvernements provinciaux, le renversement d'intérêts stratégiques est évident. Si le Québec signait un accord, la légitimité du gouvernement péquiste s'en serait trouvée renforcée et le débat politique relancé sur une base favorable au gouvernement québécois. Pour les élites politiques du Canada anglais cette situation devait être évitée a tout prix. Quand on examine de près le Canada Bill et le ridicule de certaines de ses provisions et l'absence de plusieurs autres, on doit conclure que le prix à payer a effectivement été très élevé. C'est donc dire que la determination des acteurs devait être très grande.

Pour le gouvernement Trudeau et le Parti Libéral fédéral, un accord signé aussi par le Québec aurait representé une véritable catastrophe. Au Québec, les succés électoraux de ce parti reposent en bonne partie sur la théorie des contrepoids. Avec un gouvernement péquiste ayant signé l'accord constitutionnel, il 
aurait été difficile au Parti Libéral fédéral de continuer à prétendre qu'il est la seule barrière efficace contre le séparatisme péquiste. Une analyse des calculs stratégiques des acteurs montre donc qu'au delà de la rhétorique, c'est le reste du Canada qui n'avait pas d'intérêt à s'entendre avec le Québec, et non l'inverse.

Ce n'est pas faire preuve de manichéisme que de croire que les négociateurs canadiens-anglais ont agi selon leurs intérêts. Le contraire aurait été surprenant. Pour l'instant, on ne peut qu'en prendre note. Il faudra donc que les Québécois envisagent d'autres routes pour parvenir à l'égalité politique à laquelle ils continuent d'aspirer. Jusqu'à présent, ils avaient espèré que cette reconnaissance s'obtiendrait dans le Canada (fédéralisme renouvelé) ou avec le Canada (souveraineté-association). Il faut maintenant envisager qu'elle se fasse contre le Canada (indépendance). A court terme, cela veut peut-être dire faire élire une nouvelle génération de députés à Ottawa afin de fausser encore un peu plus le jeu politique canadien ou empêcher la mise en place d'un plan canadien pour sortir le pays de la crise économique. Les Québécois et Canadiens paieront sans doute un prix élevé pour ces stratégies du pire, mais c'est la voie que semble avoir déjà choisi le reste du pays. Il ne reste plus qu'à lui emboiter le pas.

Real politik, quand tu nous tiens! 